\title{
Electroencephalographic coherence analysis in multiple sclerosis: correlation with clinical, neuropsychological, and MRI findings
} Letizia Leocani, Tiziana Locatelli, Vittorio Martinelli, Marco Rovaris, Monica Falautano,
Massimo Filippi, Giuseppe Magnani, Giancarlo Comi
Department of Clinical Neurophysiology, Scientific Institute $\mathbf{H}$ San Raffaele, Via Olgettina 60, 20132 Milan, Italy

L Leocani

T Locatelli

G Comi

Department of

Neurology

V Martinelli

M Falautano

G Magnani

G Comi

Neuroimaging

Research Unit

M Rovaris

M Filippi

G Comi

Correspondence to: Professor Giancarlo Comi g.comi@hsr.it

Received 9 November 1999 and in revised form

21 March 2000

Accepted 14 April 2000

\begin{abstract}
Objective-To explore functional corticocortical connections in multiple sclerosis by means of coherence of the EEG, and to evaluate their correlations with the degree of cognitive impairment and with brain lesion load assessed by MRI.

Methods-EEG coherence was studied from 28 patients with clinically definite multiple sclerosis. Ten minutes of resting EEG were recorded with 20 scalp electrodes, with binaural reference. FFT power and coherence were calculated in artifact free epochs of 1 second and compared with values from 22 control subjects of comparable age and sex distribution. Patients also underwent MRI $(n=27)$ and neuropsychological examination $(n=21)$.

Results-Compared with controls, patients with multiple sclerosis showed increased $\theta$ power in the frontotemporalcentral regions $(p<0.005) . \quad \theta$ Band coherence was decreased between homologous areas $(p<0.02), \alpha$ Band coherence was decreased both in the local and long distance connections $(p<0.0005)$. These findings were most striking both in patients with high MRI subcortical lesion load and in patients with cognitive involvement. A significant correlation was found between interhemispheric $\theta$ $(p=0.02)$ and $\alpha(p=0.017)$ and anteroposterior $\alpha(p=0.013)$ coherence and subcortical MRI lesion load, but not with exclusively periventricular lesion load.

Conclusions-These findings support the hypothesis that cognitive impairment in multiple sclerosis is mostly dependent on involvement of corticocortical connections related to demyelination and/or axonal loss within the white matter immediately underlying the cortex.

(F Neurol Neurosurg Psychiatry 2000;69:192-198)
\end{abstract}

Keywords: multiple sclerosis; coherence; cognitive impairment; magnetic resonance imaging

Neuropsychological assessment in patients with multiple sclerosis is receiving growing attention, due to the frequent occurrence of cognitive impairment in these patients. ${ }^{1}$ The neuropsychological pattern generally seen in multiple sclerosis consists of memory impairment, ${ }^{2}$ sustained attention deficits, visuospatial perception abnormalities, slowing of information processing, and abnormal problem solving and abstract reasoning. ${ }^{3}$ This pattern is compatible with a "subcortical" dementia..$^{3-5}$ A possible important role of the disruption of neural connections among cortical associative areas as well as between cortical and subcortical structures, occurring as a consequence of demyelination and axonal degeneration, has been suggested. ${ }^{5-7}$ Moreover, the greater importance of lesions immediately underlying the cortex with respect to other lesion locations, has been proposed. ${ }^{89}$

Coherence analysis of the EEG is a useful indicator of functional connections between different cortical areas. ${ }^{10-12}$ Coherence between two EEG signals is the spectral cross correlation normalised by their power spectra. Because coherence is normalised by the power of a given frequency band, it is independent of the amplitude of the oscillations of the two signals. This property makes coherence analysis a relatively safe method when comparing different populations, as EEG power differences between the two groups do not affect the coherence estimates. Increased coherence has been shown over the cortical or subcortical sites involved in generation and spreading of epileptic activity. ${ }^{13}{ }^{14}$ Reduced interhemispheric coherence has been reported in acallosal patients during wakefulness ${ }^{15}{ }^{16}$ and sleep. ${ }^{17}$ Decreased coherence of the resting EEG was also seen in patients with negative schizophrenic symptoms, ${ }^{18}$ Alzheimer's disease, ${ }^{19-21}$ multi-infarct dementia, ${ }^{19-21}$ and HIV/AIDS, ${ }^{23}$ and interpreted as indicating corticalfunctional disconnection in these diseases. Moreover, different patterns of EEG coherence are present in cortical and subcortical dementia. ${ }^{19}$ In this study we analysed EEG coherence in multiple sclerosis and its correlation with cognitive involvement and pattern of brain lesions assessed by MRI.

\section{Methods}

PATIENTS AND CONTROLS

Twenty eight relapse free patients (17 men, 11 women; mean age 45.4 (SD 12) years, range 20-63; mean disease duration 12.7 (SD 8.9) years, range 2-30) affected by definite multiple sclerosis of the progressive form (16 primary progressive (PP), 11 secondary progressive (SP), one transitional ( $\mathrm{Tr}$ )), according to the criteria of Lublin and Reingold ${ }^{24}$, participated in the study. Neurological disability, measured using the Kurtzke expanded disability status scale (EDSS), ${ }^{25}$ was on average 5.3 (SD 1.6) 
(range 2.5-8). Patients taking steroid treatment in the month before examination or under psychotropic drugs during the past 2 weeks were not included. Twenty two normal volunteers with similar age and sex distribution were also studied as controls. Patients also underwent MRI examination (except for one patient) and neuropsychological testing (except for seven patients). All patients and subjects gave their informed consent to participate in the study, which was approved by the local ethics committee.

MRI

Conventional spin echo dual echo $(\mathrm{TR}=2000$, $\mathrm{TE}=30 / 80$ ) brain MR scans were obtained using a $1.5 \mathrm{~T}$ machine (Siemens Magnetom SP63). Twenty four $5 \mathrm{~mm}$ thick axial contiguous slices were acquired, with a $250 \mathrm{~mm}$ field of view and a $256 \times 256$ image matrix. Patients were positioned in the scanner using published guidelines. ${ }^{26}$ For image analysis, multiple sclerosis lesions were counted on short echo, proton density (PD) weighted images (long echo T2 weighted images were used to increase the confidence in lesion detection). A scoring system $^{27}$ weighted for lesion size was used to estimate the lesion loads; one point was given
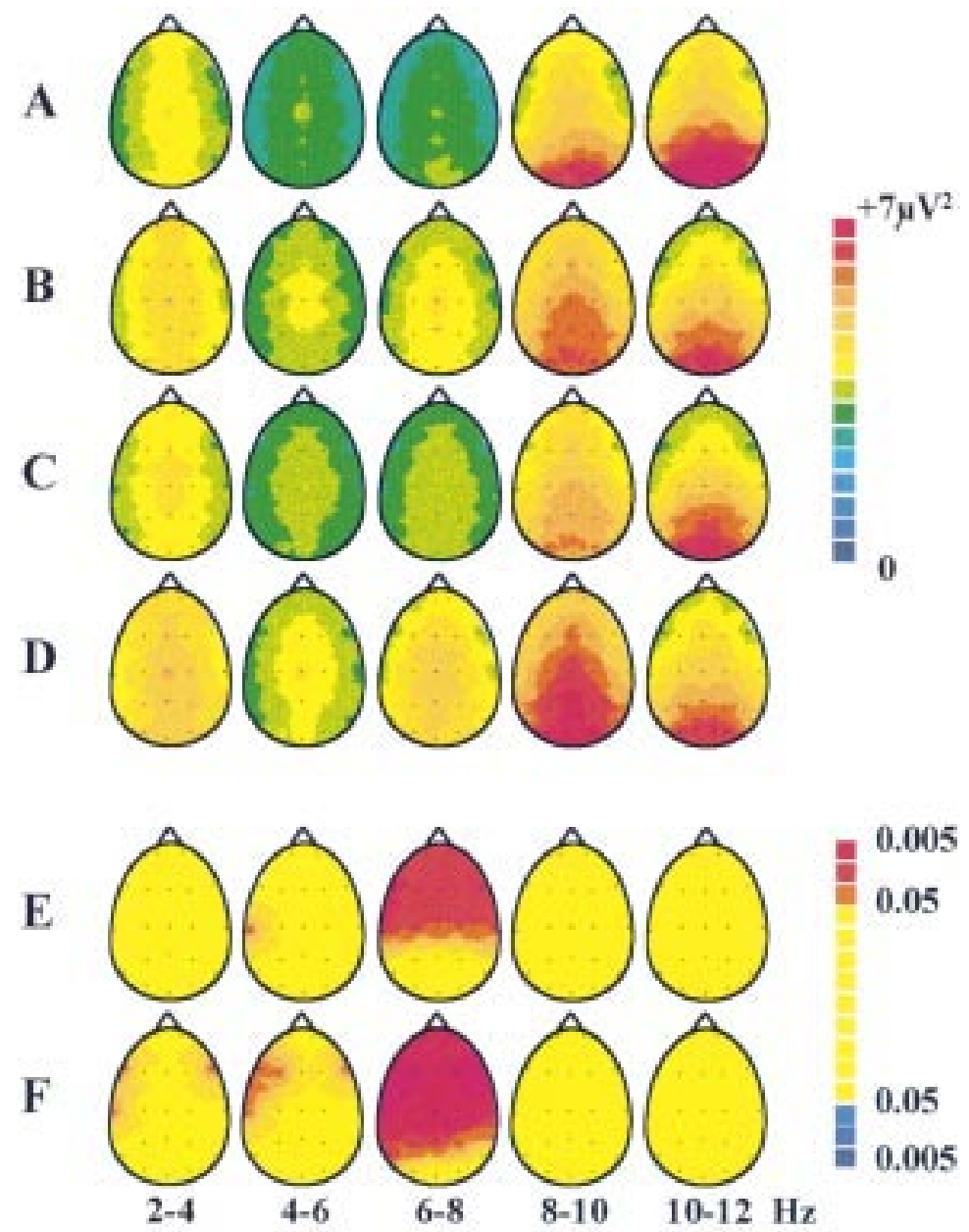

Figure 1 Group averages of absolute power maps. A: normal subjects ( $n=22)$. B: patients with multiple sclerosis who underwent cognitive tests $(n=21)$. C: cognitively unimpaired multiple sclerosis $(n=12)$. D: cognitively impaired multiple sclerosis $(n=9) . E-F: p$ values (Student's $t$ test). E: group B v group A. F: group D v group A. Red shades in E-F represent a significant power increase in groups $B$ and $D$. for each lesion with a diameter $1-5 \mathrm{~mm}$, two points for $6-10 \mathrm{~mm}$ lesions, three points for lesions greater than $10 \mathrm{~mm}$, and four points for extensive confluent lesions. Both total and regional (periventricular and subcortical) brain lesion loads were then calculated simply by multiplying the number of lesions by their individual scores. Patients were subdivided in two groups according to their median values of the total (cut off $\geqslant 60$ ) and of the subcortical (cut off $\geqslant 10$ ) lesion loads.

\section{NEUROPSYCHOLOGICAL EXAMINATION}

Patients underwent a comprehensive battery of neuropsychological tests: Wechsler adult intelligence scale (WAIS), ${ }^{28}$ Wechsler memory scale (WMS), ${ }^{29}$ digit span, ${ }^{30}$ Corsi span and Corsi supraspan, ${ }^{31}$ Raven, ${ }^{32}$ Weigl, ${ }^{33}$ Wisconsin card sorting test, ${ }^{34}$ short story, ${ }^{30}$ word pairs and word list, ${ }^{35}$ phonemic and semantic fluency, ${ }^{36}$ and attentional matrices. ${ }^{30}$ Patients were considered cognitively impaired if they failed in three or more of these tests. Nine patients out of $21(42.8 \%)$ were classified as cognitively abnormal according to this criterion.

\section{EEG RECORDING AND ANALYSIS}

Ten minutes of eye closed EEG at rest were acquired using $19 \mathrm{Ag} / \mathrm{AgCl}$ electrodes, fixed to the scalp with collodion according to the $10 / 20$ International System, with binaural reference (bp 1-35 Hz). The EEG signal was digitised (250 Hz sampling frequency) and stored for off line analysis. Artifact free epochs of 1 second were selected for calculation of fast Fourier transform power and coherence. Group comparisons of EEG absolute power were performed using the unpaired Student's $t$ test for frequencies between 2 and $20 \mathrm{~Hz}$, in steps of 1 $\mathrm{Hz}$. Coherence analysis for all electrode pairs was performed for the $\theta$ and $\alpha$ frequency bands $(\theta 4-8 \mathrm{~Hz} ; \alpha$ : $9-12 \mathrm{~Hz}$ ), as the major power differences between multiple sclerosis and control subjects were within these two bands (see Results section).

STATISTICAL ANALYSIS

We performed group comparisons of power and coherence using Student's $t$ test for unpaired data. Previous data from our group ${ }^{22}$ have shown that for each electrode pair the coherence values are distributed according to a gaussian function. The group average coherences of all patients with multiple sclerosis and control subjects were compared for each electrode pair except for the temporal electrodes, excluded from the analysis to avoid contamination from muscle artifacts. Bonferroni correction was applied to group comparisons of coherence values. Besides subdividing patients into two groups according to the presence of cognitive impairment neuropsychologically assessed, patients were also subdivided into two groups according to their total or, independently, to their subcortical MRI lesional score. For regression analysis between coherence and MRI data, coherence values for selected pairs were averaged to obtain, for each patient, a single value for anteroposterior and, separately, interhemispheric coherence. For 

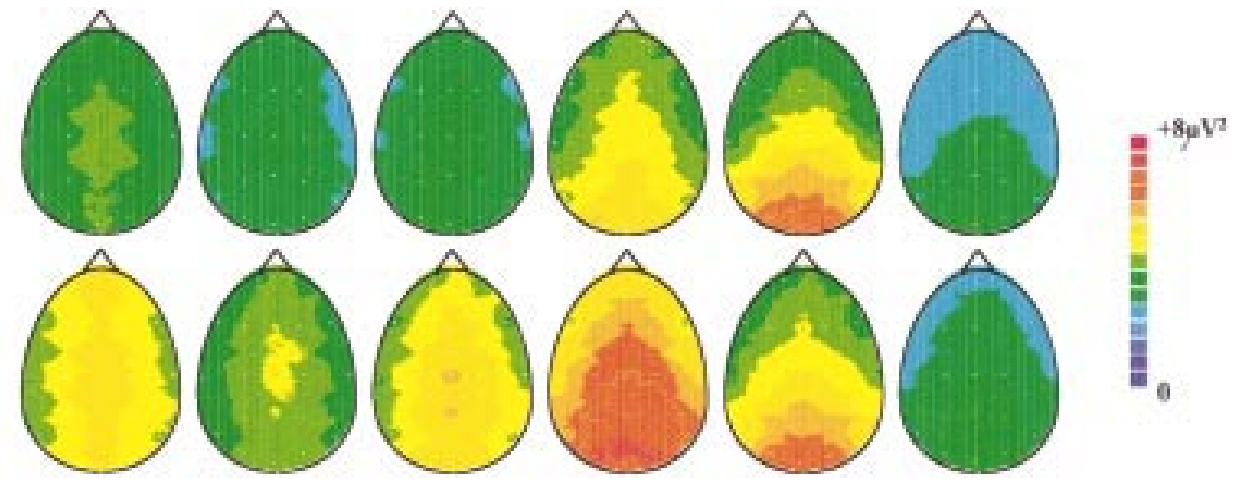

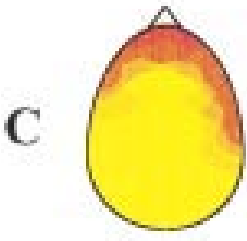

$2-4$

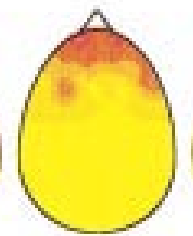

4-6

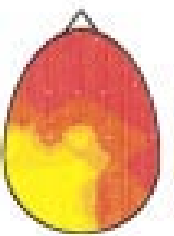

6-8

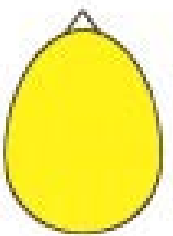

8-10

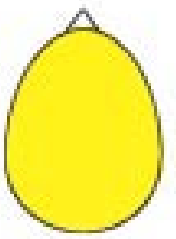

10-12
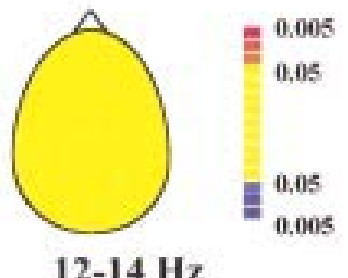

Figure 2 Group averages of absolute power maps. (A) patients with multiple sclerosis $(n=12)$ with low subcortical lesion load $(<10)$. (B) patients with multiple sclerosis $(n=15)$ with high subcortical lesion load $(\geqslant 10)$. (C) group $A v$ group $B$ ( $p$ values; Student's $t$ test). Red shades in $(C)$ represent significant power increase in group $B$.

each subject, anteroposterior coherence was the average of values from the midline pairs (FZ-CZ, FZ-PZ, FZ-OZ, CZ-PZ, CZ-OZ, $\mathrm{PZ}-\mathrm{OZ}$ ) and from pairs along the midline on the two sides (FP1-F3, FP1-C3, FP1-P3, FP1O1, F3-C3, F3-P3, F3-O1, C3-P3, C3-O1, $\mathrm{P} 3-\mathrm{O} 1$, plus the homologous pairs). For interhemispheric coherence, non-homologous pairs were considered only within the distance of a single position in the anteroposterior direction according to the 10-20 system (for example, C3-F4, C3-P4), Non- homologous pairs with distance of more than two positions in the anteroposterior direction (for example FP1C4) were not included in the average value of interhemispheric coherence, as it has been estimated that anatomical interhemispheric connections of this type are very scarce. ${ }^{37}$ Therefore, interhemispheric coherence was the average from the pairs FP1-FP2, FP1-F4, F3-FP2, F3-F4, F3-C4, C3-F4, C3-C4, C3$\mathrm{P} 4, \mathrm{P} 3-\mathrm{C} 4, \mathrm{P} 3-\mathrm{P} 4, \mathrm{P} 3-\mathrm{O} 2, \mathrm{O} 1-\mathrm{P} 4, \mathrm{O} 1-\mathrm{O} 2$.

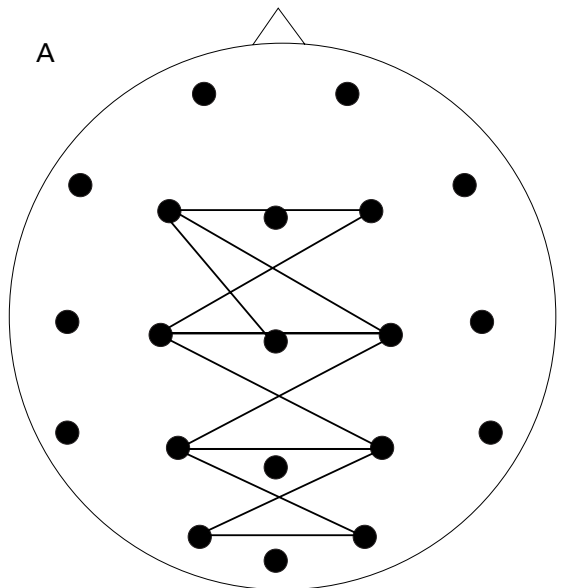

\section{Results}

POWER

Considered as a group, patients with multiple sclerosis had a significant increase of $\theta$ power in the frontotemporal-central regions compared with normal subjects $(\mathrm{p}<0.05$ in all frontal and central electrodes, T3 and T4; fig 1). In fig1, only patients who underwent neuropsychological evaluation were considered $(n=21)$. A similar pattern was found in patients with cognitive impairment ( $n=9$; five $P P$, four $S P$ ) if compared both with normal subjects $(\mathrm{p}<0.02$ at all frontal, central, T3, and T4 electrodes; $p<0.05$ in all but occipital and T6 electrodes; fig 1) and to cognitively normal patients $(n=12$; seven $\mathrm{PP}$, five SP) but the latter comparison did not reach statistical significance. Cognitively normal patients had no power difference from normal controls. No significant power difference was found when comparing patients with a high $(n=17$; nine PP, eight $S P)$ versus a low $(n=10$; six $\mathrm{PP}$, three $\mathrm{SP}$, one $\mathrm{Tr}$ ) total lesion load on

Figure 3 Coherence maps. Lines represent significant $(p<0.05)$ coherence decrease in the group of patients with multiple sclerosis who underwent cognitive tests $(n=21)$ compared with normal subjects $(n=22)$. Temporal electrodes were excluded from the analysis. (A) $\theta(4-8 \mathrm{~Hz}) ;(B) a(9-12 \mathrm{~Hz})$.

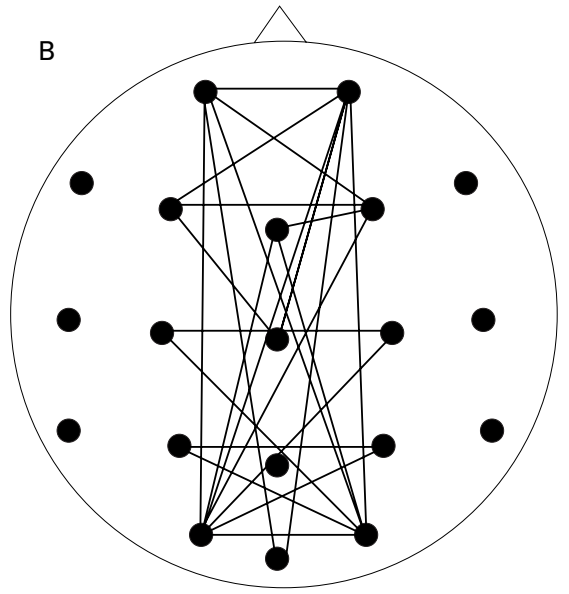



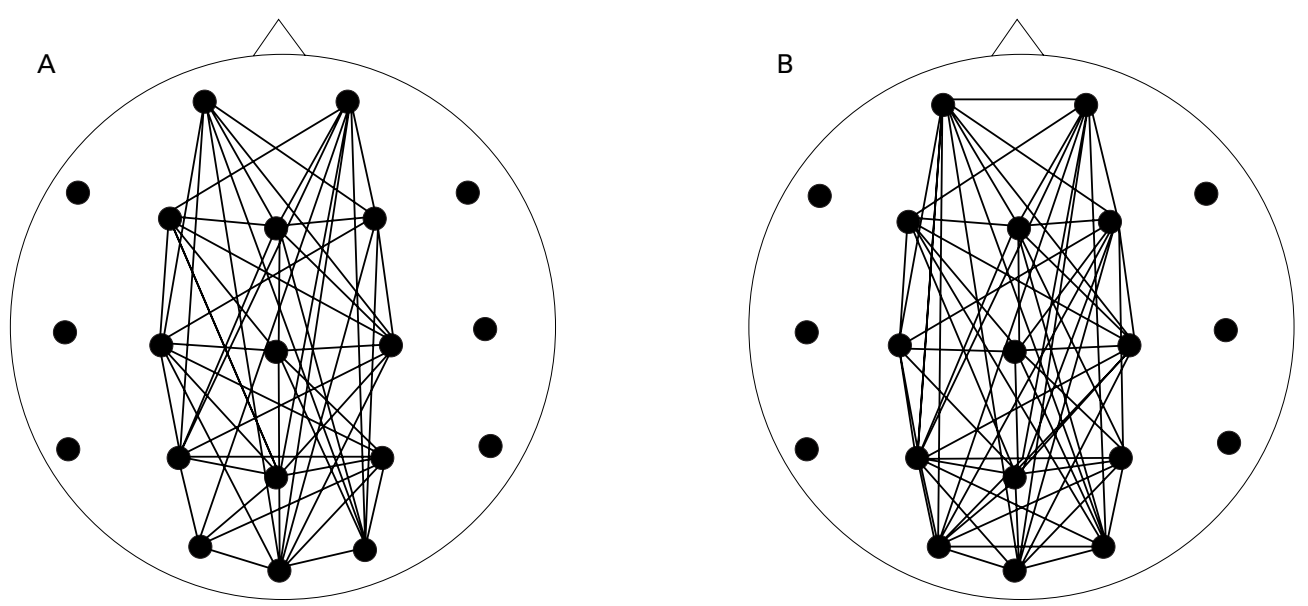

Figure 4 Coherence maps. Lines represent significant $(p<0.05)$ coherence decrease in the group of cognitively impaired $(n=9)$ compared with cognitively normal $(n=12)$ patients with multiple sclerosis. Temporal electrodes were excluded from the analysis. (A) $\theta(4-8 \mathrm{~Hz}) ;(B) a(9-12 \mathrm{~Hz})$.

MRI, whereas a significant increase of $\delta$ ( $<<0.05$ at electrodes FP1, FP2, F7, F8, T4) and $\theta(\mathrm{p}<0.05$ at all frontal, C3, C4, P4, T6, O1 electrodes) was present in patients with high ( $n=15$; eight $P P$, seven $S P$ ) compared with low ( $n=12$; seven PP, four SP, one Tr) subcortical lesion load (fig 2). Patients with memory impairment ( $n=4$, two PP, two SP) had a higher power in the $\delta$ and $\theta$ bands over the left frontotemporal electrodes than normal subjects (data not shown; $\mathrm{p}<0.02$ at electrodes F7, $\mathrm{F} 3, \mathrm{Fz}, \mathrm{T} 3, \mathrm{C} 3, \mathrm{~F} 8, \mathrm{~T} 4$ for $\delta$ and at all but occipital electrodes for $\theta$ ) and the other patients $(\mathrm{p}<0.05$ at FP1, FP2, F3, C3, Cz for $\delta$ and $\mathrm{Fz}$ for $\theta$ ).

COHERENCE

Compared with controls, patients with multiple sclerosis had reduced coherence of both $\theta$ and $\alpha$ bands ( $p$ between 0.05 and 0.002 for the $\theta$ band; $p<0.0005$ for the $\alpha$ band, significant if Bonferroni corrected; figure 3 ). In this figure, only patients who underwent neuropsychological examination were considered $(n=21)$. $\theta$ Band coherence was reduced mostly between
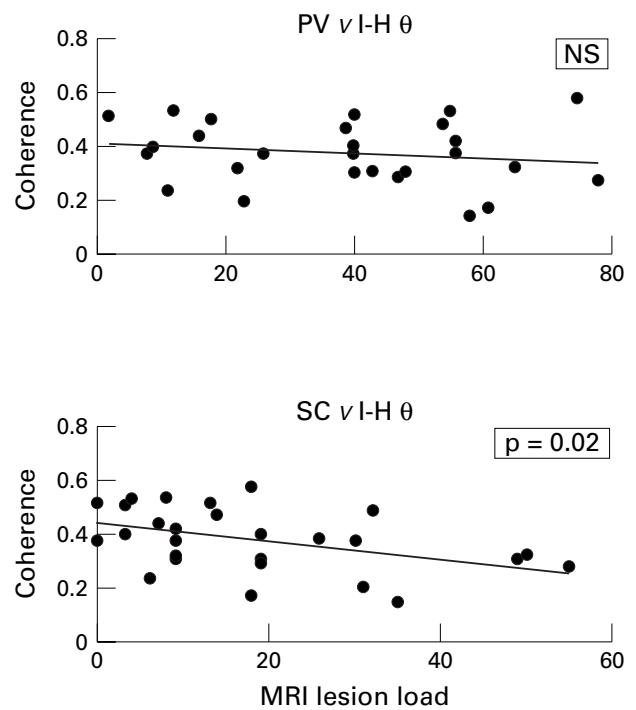

interhemispheric homologous electrodes or within the distance of a single position in the anteroposterior direction (P3-C4; P3-O1). $\alpha$ Band coherence was diffusely decreased both between interhemispheric and anteroposterior electrode pairs (figure 3). Cognitively impaired patients (figure 4) had a significant diffuse coherence decrease of the two bands compared with cognitively normal patients, in the anteroposterior and in the interhemispheric direction $(p<0.0005$, significant if Bonferroni corrected).

\section{CORRELATIONS BETWEEN COHERENCE AND MRI}

DATA

No significant correlation was present between coherence and periventricular lesion load, whereas subcortical lesion load was significantly correlated with interhemispheric $\theta$ and $\alpha$ coherence $(p=0.020$ and $p=0.017$ respectively, figure 5) and with anteroposterior $\alpha$ coherence $(p=0.013)$. The total lesion load had a correlation at the limit of statistical significance $(p=0.05)$ only with the interhemispheric $\alpha$ band coherence.
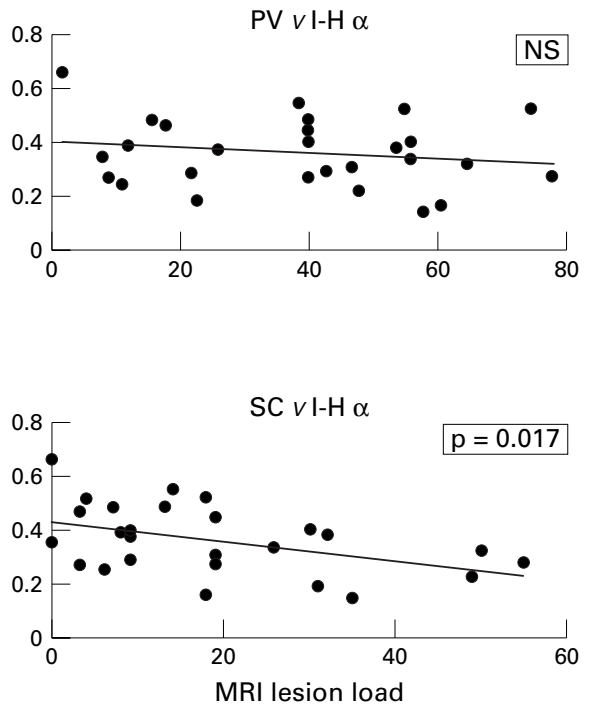

Figure 5 Regression of interhemispheric (I-E) coherence with periventricular (PV) and subcortical (SC) MRI lesion load in each patient with multiple sclerosis $(n=27)$. 


\section{Discussion}

In this study we evaluated computerised EEG spectral power and coherence in patients with multiple sclerosis, together with neuropsychological tests and brain MRI.

EEG POWER

We found a slowing of the background EEG activity in patients with multiple sclerosis compared with control subjects, together with a diffuse increase of slow oscillations in the anterior and temporal regions. Moreover, patients with memory impairment had an increase of slow components $(\delta$ and $\theta)$ in the left temporofrontal regions. Compared with normal subjects, power abnormalities were significant only in the group of patients with cognitive impairment and not in cognitively normal patients. Considering MRI, the groups of patients with high and low total lesion load had no significantly different EEG power from normal subjects and from each other. Conversely, patients with high subcortical lesion load had more $\theta$ and $\delta$ power compared with normal controls and with patients with low subcortical lesion load, who had normal EEG considered as a group. Other studies reported EEG abnormalities in multiple sclerosis, ${ }^{38-41}$ mainly increased slow frequencies and reduced $\alpha$ band. Computerised spectral analysis ${ }^{38}$ showed a relation between disability and the amount of $\beta$ activity in the frontocentral area and of $\theta$ activity in the temporal area. Moreover, after improvement due to immunosuppressive treatment, a marked increase of mean $\alpha$ frequency in the parieto-occipital region was present. Quattrini $e t a l^{39}$ in a 5 year follow up study, did not find a significant correlation between visual EEG abnormalities and neurological or psychic status, but there was a weak correlation between EEG and CT findings. Facchetti et alt failed to find a significant correlation between conventional or quantitative EEG and MRI findings or disability. Feng, ${ }^{41}$ at visual inspection of the EEG, found more abnormal records in active than quiescent patients with multiple sclerosis; moreover, patients with relapses had more frequent abnormalities than patients with a progressive course. In the present study, we investigated patients with a progressive form of multiple sclerosis, in which a higher prevalence of cognitive disturbances, compared with patients with relapsing-remitting disease, has been reported. ${ }^{42}{ }^{43}$ Whether patients with secondary progressive disease are more affected by cognitive impairment than primary progressive ones is still a controversial issue, as this has been found by some authors ${ }^{44}$ and denied by others. ${ }^{46}$ Recent evidence, ${ }^{46}$ however, indicates that, when patients with primary or secondary progressive multiple sclerosis have similar disability and MRI lesion burden, the frequency and severity of cognitive disturbances do not differ significantly between these two multiple sclerosis phenotypes. This might explain why, in our study, the groups of patients with and without cognitive impairment were not clustered for either form of progressive multiple sclerosis. Our findings show that lesions immediately underlying the cortex, among other lesion locations, are the major determinant of the EEG power abnormalities encountered in multiple sclerosis. Several studies ${ }^{45} 48$ have shown a statistically significant correlation between the global cognitive impairment and the severity of white matter abnormalities of the hemispheres, of corpus callosum atrophy, and of ventricular dilatation. More strict correlation has been found between cognitive impairment and both subcortical lesion $\operatorname{load}^{89}$ and corpus callosum atrophy. ${ }^{49}$ Moreover, the analysis of regional cerebral lesion load showed significant relations with specific cognitive functions. ${ }^{48-50}$

EEG COHERENCE

Our study has demonstrated a significant decrease of $\alpha$ and $\theta$ band coherence between both anteroposterior and interhemispheric areas in patients with progressive multiple sclerosis. This reduction was significant in cognitively impaired patients, both compared with normal subjects and with cognitively normal patients with multiple sclerosis, who showed no statistically significant coherence difference with normal controls. As coherence is considered as an indicator of functional neuronal connections between different cortical areas, ${ }^{1012}$ a coherence decrease between two regions would indicate a decrease in their functional connections. Nevertheless, other factors could affect coherence estimates. Decreased coherence between $\mathrm{O} 1$ and $\mathrm{O} 2$ leads for the $\alpha-1$ band and between F3 and F4 for the $\beta-1$ band has also been shown in normal subjects during light drowsiness compared with the awake state. ${ }^{51}$ In these findings, the decreased interhemispheric coherence for those bands was accompanied by increased coherence within the $\theta-1$ and $\beta-1$ bands between $\mathrm{C} 4$ and $\mathrm{O} 2$. Even though we excluded through visual inspection EEG segments with signs of decreased arousal, it is possible that some decrease in interhemispheric coherence may be due to decreased arousal not evident at visual inspection of the EEG. Nevertheless, in our study the $\theta$ band coherence was also decreased in patients with multiple sclerosis with cognitive impairment compared both with normal subjects and with patients with multiple sclerosis without cognitive impairment. Interhemispheric coherence for both bands was also inversely correlated with subcortical lesion load. Therefore, increased drowsiness in deteriorated patients cannot totally account for our findings.

Another problem arising when interpreting coherence differences between groups is the reference used. Using a common reference (linked ears) a signal with high power ( $\alpha$ oscillations) may produce an artificially high coherence between distant electrodes because of volume conduction. Moreover, the reference electrodes may record brain activity and project it to the EEG channels, thus artificially increasing the coherence estimates, as reported by Fein et $a .^{52}$ If this were the case in our study, it could be possible that normal subjects, having a higher $\alpha$ power than patients with multiple sclerosis, display a higher $\alpha$ coherence 
because of volume conduction of the oscillations. A method for reducing the signal introduced by the reference is the transformation of the data using the Laplacian operator ${ }^{53-55}$ although an agreement about the optimal computational algorithm is not completely reached. ${ }^{56-59}$ For our coherence results, obtained using a common reference recording, if the finding of decreased $\alpha$ band coherence in patients with multiple sclerosis would be due to an artificially high $\alpha$ coherence in the control subjects (who had a higher $\alpha$ power), an increased $\theta$ coherence in patients with multiple sclerosis compared with controls would be expected, as $\theta$ power was higher in patients with multiple sclerosis. This was not the case in our findings, as both $\alpha$ and $\theta$ band coherence were lower in patients with multiple sclerosis compared with controls. Thus our results cannot be explained by an artificially high coherence caused by the use of a linked ear reference.

In the present study we found a significant correlation between anteroposterior coherence and the lesion load immediately underlying the cortex ("subcortical"). It has been reported that cognitive impairment is mostly related to lesions of the white matter immediately underlying the cortex, but not with exclusively periventricular lesions. ${ }^{89}$ These lesions may produce a deafferentation of cortex from underlying structures, but also involve long corticocortical projections. The superior longitudinal fasciculus and the corpus callosum are good candidates for anteroposterior and interhemispheric connections, respectively. Nevertheless, their importance in determining functional connections reflected in EEG coherence has not been clearly determined. Although studies specifically involving the superior longitudinal fasciculus and EEG coherence are missing, contrasting results have been obtained concerning the corpus callosum. Lesions or agenesis of the corpus callosum have been reported to leave the degree of synchronisation between the two hemispheres unaffected ${ }^{6061}$ or decreased..$^{15-1762}$ Residual posterior interhemispheric coherence in acallosal patients has been attributed to the persistence of the posterior commissure, ${ }^{17}$ moreover, the pattern of decreased coherence has been found to be consistent with the topography of the surgical section. ${ }^{62}$

Another contribution to EEG coherence between different cortical areas can be provided by synchronisation from a common pacemaker, such as the thalamus. It may be hypothesised that if the interruption of thalamic projections to the cortex is the major determinant of decreased coherence in our findings, coherence should be more highly correlated with periventricular lesions than with subcortical lesions immediately underlying the cortex. In fact, whereas the former lesions would be most likely to involve thalamocortical connections, the latter lesions would also involve long corticocortical projections. In our findings, there was a high correlation between EEG coherence and lesion load in the white matter close to the cortex, whereas no correla- tion was present with periventricular lesions. These findings are also consistent with anatomical studies estimating that thalamic fibres entering the cerebral cortex are only up to $1 \%$ of the total, ${ }^{63-64}$ even though we should consider that the extent of anatomical representation does not necessarily correspond to functional relevance. Indeed, it cannot be excluded that the mechanism by which subcortical lesions may affect coherence may also be mediated by effects on the thalamus. Contreras et $a l^{64}$ have recently demonstrated that, in anaesthetised cats, the synchronisation of thalamic sleep spindles between different sites in the thalamus is dependent on the integrity of the overlying cortex. Moreover, the coherence between sleep spindles at different electrodes of an array placed over the suprasylvian cortex in the anteroposterior direction, was not disrupted by a coronal cut through the suprasylvian gyrus. The authors commented that it is unlikely that coherence was unaffected because of other intact corticocortical connections, as the same cut disrupted coherence of a slow oscillation generated intracortically. ${ }^{65}$ It has to be said that these studies were performed on sleep spindles and not on the $\alpha$ and $\theta$ rhythms. Nevertheless, it is still possible that the white matter lesions close to the cortex, correlating with decreased EEG coherence in multiple sclerosis, may affect not only the corticocortical connections and the thalamocortical fibres for the overlying cortex, but also reduce the cortical output to the thalamus. According to Contreras et $a l^{64}$ a lesion of corticothalamic fibres would lead to a decreased coherence of the intrinsic thalamic oscillations. Therefore, different thalamic regions would drive the cortical oscillations through those thalamocortical fibres which have remained intact, in a less coherent manner.

In conclusion, slowed EEG background activity and reduced coherence were present both in cognitively impaired patients compared with unimpaired patients and in patients with high compared with low subcortical MRI lesion load. Moreover, subcortical lesion load correlated highly with reduction of EEG coherence. Taken altogether, these data are consistent with the hypothesis that cognitive dysfunction in multiple sclerosis is dependent on the disconnection of cortical associative areas, produced by demyelination, or axonal loss, or both, in the subcortical white matter occurring in these patients.

We are grateful to Aldo Elia for the EEG recordings. This study was supported by a grant from the Istituto Superiore di Sanità (National Ministry of Health, Rome, Italy; protocol number 96/J/T44).

1 Peyser JM, Rao SM, Larocca NG, et al. Guidelines for neuropsychological research in multiple sclerosis. Arch Neurol 1990;47:94-7.

2 Beatty WW. Memory and frontal lobe dysfunction in multiple sclerosis. F Neurol Sci 1993;115 (suppl):S38-41.

3 Rao SM. Neuropsychology of multiple sclerosis: a critical review. f Clin Exp Neuropsychol 1986;5:503-42.

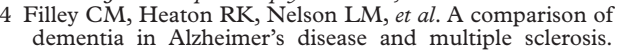
dementia in Alzheimer's disea

5 Comi G, Filippi M, Martinelli V, et al. Brain magnetic resonance imaging correlates of cognitive impairment in multiple sclerosis. F Neurol Sci 1993;115:66-73. 
6 Rao SM. Multiple sclerosis. In: Cummings JL, ed. Subcortical dementia. New York, NY: Oxford University Press,

7 Mahler ME, Benson DF. Cognitive dysfunction in multiple sclerosis: a subcortical dementia? In: Rao SM, ed. Neurobehavioral aspects of multiple sclerosis. New York, NY: Oxford University Press, 1990:88-101.

8 Damian multiple sclerosis, Schilling G, Bachmann G, et al. White matter lesions and cognitive deficits: relevance of lesion pattern? Acta Neurol Scand 1994;90:430-6.

9 Miki Y, Grossman RI, Udupa JK, et al. Isolated U-fiber involvement in multiple sclerosis: preliminary observations. Neurology 1998;50:1301-6.

10 Sklar RL, Hanley J, Simmons WW. An EEG experiment aimed toward identifying dyslexic children. Nature 1972,240:414-16.

11 Busk G, Galbraith GC. EEG correlates of visual motor practice in man. Electroencephalogr Clin Neurophysiol 38:415-22.

12 Thatcher RW, Krause PJ, Hrybyk M. Cortico-cortical associations and EEG coherence: a two-compartmental model. Electroencephalogr Clin Neurophysiol 1986;64:123-43.

13 Duckrow RB, Spencer SS. Regional coherence and the transfer of ictal activity during seizure onset in the media temporal lobe. Electroencephalogr Clin Neurophysiol 1992;82: 415-22.

14 Gotman J, Levtova V. Amygdala-hippocampus relationships in temporal lobe seizures: a phase-coherence study. Epilepsy Res 1996;25:51-7.

15 Koeda T, Knyazeva M, Njiokiktjien C, et al. The EEG in acallosal children. Coherence values in the resting state: left hemisphere compensatory mechanism? Electroencephalog Clin Neurophysiol 1995;95:397-407.

16 Nagase Y, Terasaki O, Okubo Y, et al. Lower interhemispheric coherence in a case of agenesis of the corpus callo-
sum. Clin Electroencephalogralogr 1994;25:36-9.

17 Nielsen T, Montplaisir J, Lassonde M. Decreased interhemispheric EEG coherence during sleep in agenesis of the hemispheric EEG coherence during sleep in

18 Merrin EL, Floyd TC. Negative symptoms and EEG in schizophrenia: a replication. Schizophr Res 1996;19:15161

19 Leuchter AF, Newton TF, Cook IA, et al. Changes in brain functional connectivity in Alzheimer's type and multiinfarct dementia. Brain 1992;115:1542-61.

20 Dunkin JJ, Leuchter AF, Newton TF, et al. Reduced EEG coherence in dementia: state or trait marker? Biol Psychiatry 1994;35:870-9.

21 Sloan EP, Fenton GW, Kennedy NS, et al. Neurophysiology and SPECT cerebral blood flow patterns in dementia. Electroencephalogr Clin Neurophysiol 1994;91:163-70.

22 Locatelli $\mathrm{T}$, Cursi $\mathrm{M}$, Liberati D, et al. EEG coherence in Alzheimer's disease. Electroencephalogr Clin Neurophysiol 1998;106:229-37.

23 Newton TF, Leuchter AF, Miller EN, et al. Quantitative EEG in patients with AIDS and asymptomatic HIV infection. Clin Electroencephalogralogr 1994;25:18-25.

24 Lublin FD, Reingold SC. Defining the clinical course of multiple sclerosis: results of an international survey. Neurology 1996;46:907-11.

25 Kurtzke JF. Rating neurological impairment in multiple sclerosis: an expanded disability status scale (EDSS). Neurology 1983;33:1444-52.

26 Miller DH, Barkhof F, Berry I, et al. Magnetic resonance imaging in monitoring the treatment of multiple sclerosis: concerted action guidelines. F Neurol Neurosurg Psychiatry 1991;54:683-8.

27 Ormerod IEC, Miller DH, McDonald WI, et al. The role of NMR imaging in the assessment of multiple sclerosis and isolated neurological lesions. A quantitative study. Brain 1987;110:1579-616.

28 Wechsler D. Scala di intelligenza WAIS per adulti. In: FG Ferradini, A Vassena, eds. Translation and revision. Florence: OS Organizzazioni Speciali, 1974

29 Wechsler D. Wechsler memory scale: forma 1. Florence: OS Organizzazioni Speciali, 1963.

30 Spinnler H, Tognoni G. Standardizzazione e taratura italiana di test neuropsicologici. Ital $\mathcal{f}$ Neurol Sci 1987; 8(suppl 6):1-120.

31 De Renzi E, Nichelli P. Verbal and non-verbal short term memory impairment following hemispheric damage. Cortex 1975;11:33-41

32 Raven J.C. Standard progressive matrices: sets $A, B, C, D$, (and $E), H, K$. London: Lewis, 1938

33 Weigl E. On the psychology of the so-called processes of abstraction, [translated]. Fournal of Abnormal Social Psychology 1927;36:3-33.

34 Heaton RK. Wisconsin card sorting test (manual). Odessa, FL: Psychological Assessment Resources, 1981.

35 Novelli G, Papagno C, Capitani E, et al. Tre test clinici di memoria verbale a lungo termine: taratura su soggetti normali. Archivio Neurologia Psicologia e Psichiatria 1986;47: 278-96.

36 Novelli G, Papagno C, Capitani E, et al. Tre test clinici di ricerca e produzione lessicale: taratura su soggetti normali. Archivio Neurologia Psicologia e Psichiatria 1986;47:477506.
37 Szentàgotai J. The neural network of the cerebral cortex: a functional interpretation. Proc R Soc Lond 1978;201:21948

38 Colon E, Hommes OR, de Weerd JP. Relation between EEG and disability scores in multiple sclerosis. Clin Neurol Neurosurg 1981;83:163-8.

39 Quattrini A, Paggi A, Ortenzi A, et al. CT and EEG investigations in 100 patients with multiple sclerosis (MS). Ital f Neurol Sci 1981;2:25-34.

40 Facchetti D, Mai R, Colombo A, et al. Limited clinical significance of traditional and quantitative EEG in multiple sclerosis. Acta Neurol Belg 1994;94:245-50.

41 Feng YK. Clinico-electroencephalogralografic studies of multiple sclerosis. Clin Exp Neurol 1981;17:47-57.

42 Heaton RK, Nelson LM, Thompson DS, et al. Neuropsychological findings in relapsing remitting and chronicprogressive multiple sclerosis. I Consult Clin Psychol 1985;53:103-10.

43 Beatty WW, Goodkin DE, Monson N, et al. Cognitive disturbances in patients with relapsing remitting multiple sclerosis. Arch Neurol 1989;46:1113-9.

44 Comi G, Filippi M, Martinelli V, et al. Brain magnetic resonance correlates of cognitive impairment in primary and secondary progressive multiple sclerosis. $\mathcal{F}$ Neurol Sci 1995; 132:222-7.

45 Rao SM, Leo GJ, Haughton VM, et al. Correlation of magnetic resonance imaging with neuropsychological testing in multiple sclerosis. Neurology 1989;39:161-6.

46 Foong J, Rozewicz L, Chong WK, et al. A comparison of neuropsychological deficits in primary and secondary progressive multiple sclerosis. f Neurol 2000;247:97-101.

47 Franklin GM, Nelson LM, Filter CM, et al. Cognitive loss in multiple sclerosis. Arch Neurol 1989;46:62-167.

48 Swirsky-Sacchetti T, Mitchell DR, Seward J, et al. Neuropsychological and structural brain lesions in multiple sclerosis: a regional analysis. Neurology 1992;42:1291-5.

49 Foong J, Rozewicz L, Quaghebeur G, et al. Executive functions in multiple sclerosis. The role of frontal lobe pathology. Brain 1997;120:15-26.

50 Rovaris M, Filippi M, Falautano M, et al. Relationship between MR abnormalities and patterns of cognitive impairment in multiple sclerosis. Neurology 1998;50:1601-

51 Wada Y, Nanbu Y, Koshino Y, et al. Inter- and intrahemispheric EEG coherence during light drowsiness. Clin Electroencephalogralogr 1996;27:84-8.

52 Fein G, Raz J, Brown FF, et al. Common reference coherence data are confounded by power and phase effects. Electroencephalogr Clin Neurophysiol 1988;69:581-4.

53 Hjorth B. An on-line transformation of EEG scalp potentials into orthogonal source derivations. Electroencephalogr Clin Neurophysiol 1975;39:526-30.

54 Pascual-Marqui RD, Gonzales-Andino SL, Valdes-Sosa PA, et al. Current source density estimation and interpolation based on the spherical harmonic Fourier expansion. Int $\mathcal{f}$ Neurosci 1988;43:237-49.

55 Perrin F, Pernier J, Bertrand O, et al. Spherical splines for scalp potential and current density mapping. Electroencephalogr Clin Neurophysiol 1989;72:184-7.

56 Biggins CA, Fein G, Raz J, et al. Artifactually high coherences result from using spherical spline computation of scalp current density. Electroencephalogr Clin Neurophysiol 1991;79:413-19.

57 Biggins CA, Ezekiel F, Fein G. Spline computation of scalp current density and coherence: a reply to Perrin [letter to editor]. Electroencephalogr Clin Neurophysiol 1992:83:172-4.

58 Pascual-Marqui RD. The spherical spline Laplacian does not produce artifactually high coherencies: comments on two articles by Biggins et al. Electroencephalogr Clin Neurophysiol 1993;87:62-4

59 Biggins CA, Fein G. Spline computation of scalp current density and coherence: a reply to Pascual-Marqui [letter to editor]. Electroencephalogr Clin Neurophysiol 1993;87:65-6.

60 Green JB, Russell DJ. Electroencephalographic asymmetry with midline cyst and deficient corpus callosum. Neurology 1966;16:541-5.

61 Corsi-Cabrera M, Trias G, Guevara MA, et al. EEG interhemispheric correlation after callosotomy: one case study. Percept Motor Skills 1995;80:504-6.

62 Montplaisir J, Nielsen T, Cote J, et al. Interhemispheric EEG coherence before and after partial callosotomy. Clin Electroencephalogr 1990;21:42-7.

63 Braitenberg V. Cortical architectonics: general and areal. In: Brazier MAB, Petsche H, eds. Architectonics of the cerebral cortex. New York: Raven, 1978:443-65.

64 Contreras D, Destexhe A, Sejnowski TJ, et al. Control of spatiotemporal coherence of a thalamic oscillation by corticothalamic feedback. Science 1996;274:771-4.

65 Amzica F, Steriade M. Disconnection of intracortical synaptic linkages disrupts synchronisation of a slow oscillation. $\mathcal{F}$ Neurosci 1995;15:4658-77. 